\title{
Research on Improved PID Control Algorithm of a Kind of High Speed Vehicle
}

\author{
Wenguang Zhang, Junwei Lei and Zijian Lin \\ Department of control engineering, Naval Aeronautical and Astronautical University, Yantai, 264001, \\ China \\ leijunwei@126.com
}

Keyword: PID, Stability, Robustness, Hypersonic Vehicle

\begin{abstract}
According to the nonlinear model of pitch channel of a hypersonic vehicle, the open-loop control is studied, and the response curve is given. On this basis, this paper makes a research on PID control, PID controller design and the introduction of the angular velocity to increase the system damping, which constitutes improved PID control. At the same time, single channel flight simulation is carried out, and the results of digital simulation are given.
\end{abstract}

\section{Introduction}

Since the concept of hypersonic combustion was put forward in 1950s, the research on hypersonic control in the United States and the western countries has lasted for more than half a century. In China, the research of hypersonic aircraft and superb engineering is a key project which has concentrated our much focus. It's as difficult as the spacecraft and the moon program. And Hypersonic control problem is also a hot and difficult problem in the control field at home and abroad in the last ten years. As academician Huang Lin pointed out, the strong coupling of hypersonic vehicle, classical nonlinear and fast time-varying characteristics have brought great challenges to the control of science. So it is no doubt that the research on the control theory of hypersonic vehicle is of great significance. There are a lot of researches about super hypersonic vehicle, and here we use the model of Zhou Chuan from BIT and Jiang Bin from CZ to carry on the simulation experiment.

\section{Model Description}

Considering the elastic shape structure, a kind of pitch channel hypersonic aircraft model built according to Lagrange equation is released by USA air force as followed:

$$
\begin{gathered}
\dot{V}=\frac{T \cos \alpha-D}{m}-g \sin \gamma \\
\dot{\phi}=-2 \zeta \omega_{n} \phi-\omega_{n}^{2} \phi+\omega_{n}^{2} \phi_{c} \\
\dot{\gamma}=\frac{L+T \sin \alpha}{m V}-\frac{g \cos \gamma}{V} \\
\dot{\alpha}=q-\dot{\gamma} \\
\dot{q}=\frac{M}{I} \\
\dot{h}=V \sin \gamma \\
\ddot{\eta}_{i}=-2 \varepsilon_{m} \omega_{m i} \dot{\eta}_{i}-\omega_{m i}^{2} \eta_{i}+N_{i}
\end{gathered}
$$

Where

$$
T=\bar{q} s\left(C_{T \phi} \phi+C_{T}+C_{T}^{\eta} \eta\right), \quad D=\bar{q} S C_{D}
$$

And $V$ is speed, $\gamma$ is the speed angle, $\alpha$ is attack angle, $Q$ is the attitude angle speed, $h$ is the height, $\phi$ is the oil supplying factor, $\delta_{c}$ is the duck wing and $\delta_{e}$ is the lift rudder. 


\section{Analysis of Open Loop Response}

According to the simulation results of the open-loop response of the hypersonic vehicle, the rudder deflection $\delta_{c}=0$ is set, as shown in the Matlab language program:

\section{deta=upitch;}

deta $=0$;

At this time, the control amount upitch=0, which doesn't work. And the angle of attack simulation images are as follows:

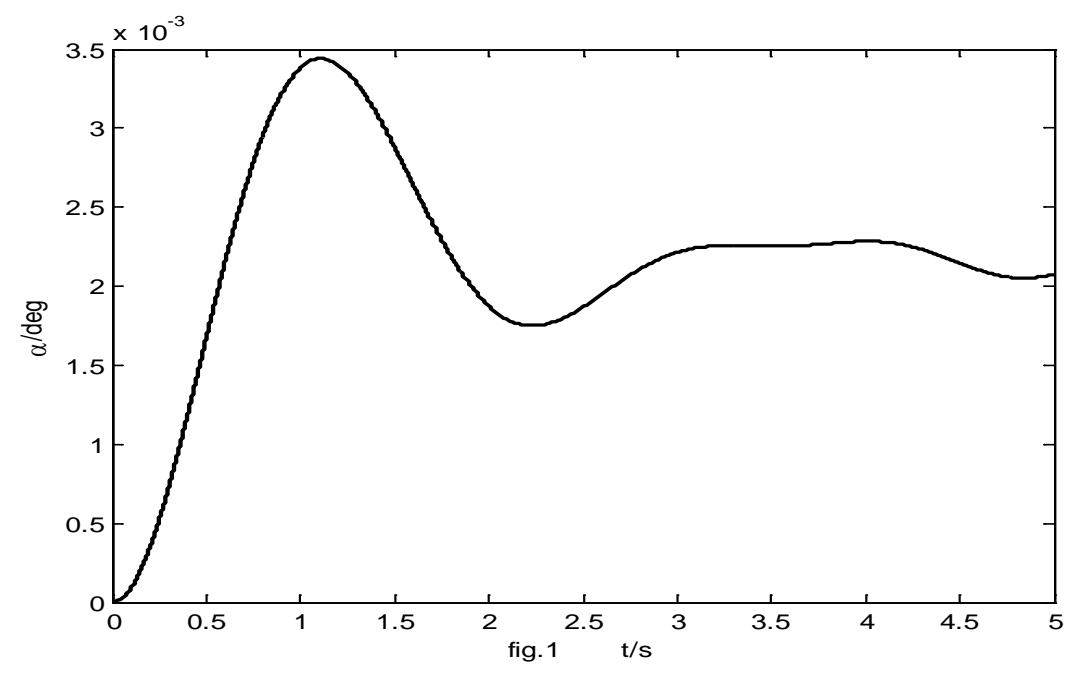

Fig. 1 Angle of attack curve

It can be seen that the angle of attack simulation image oscillation, but still is able to maintain stability. Next, we will try to add the PID control to improve the performance of the system.

The design of attack angle tracking PID controller and the definition of angle of attack error is as follows:

$$
e_{\alpha}=\alpha-\alpha^{d}
$$

The standard PID controller can be written as follows:

$$
u(t)=k_{p}\left(e(t)+\frac{1}{T_{I}} \int_{0}^{t} e(t) d t+\frac{T_{d} e(t)}{d t}\right)
$$

By introducing angular rate damping, in this paper, the improved PID controller is as follows:

$$
u_{p}=k_{p} e_{\alpha}+k_{i} \int e_{\alpha} d t+k_{d} \dot{e}_{\alpha}+k_{q} q
$$

According to the above formula, the preparation of the Matlab program is as follows:

upitch=kp_alfa*ealfa+ks_alfa*sealfa+kd_alfa*dalfa+q*kq;

deta=upitch;

Finally, based on the above model, the single channel flight simulation program is established, and the flight simulation curve is obtained as follows:

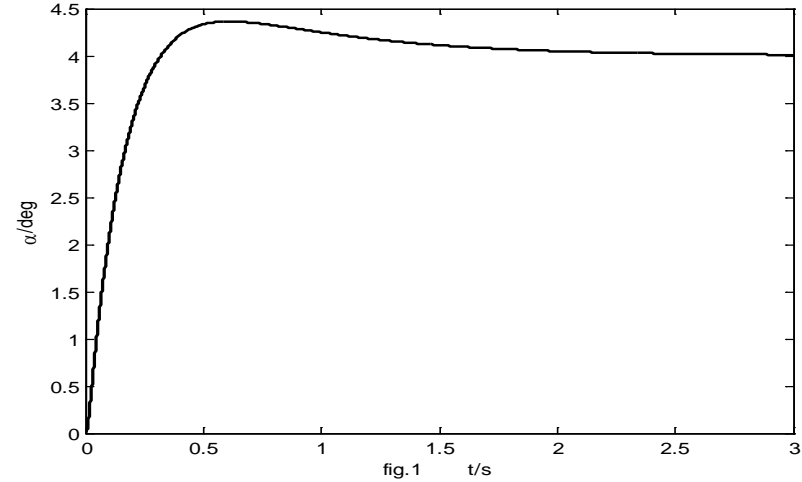

Fig. 2 Angle of attack curve

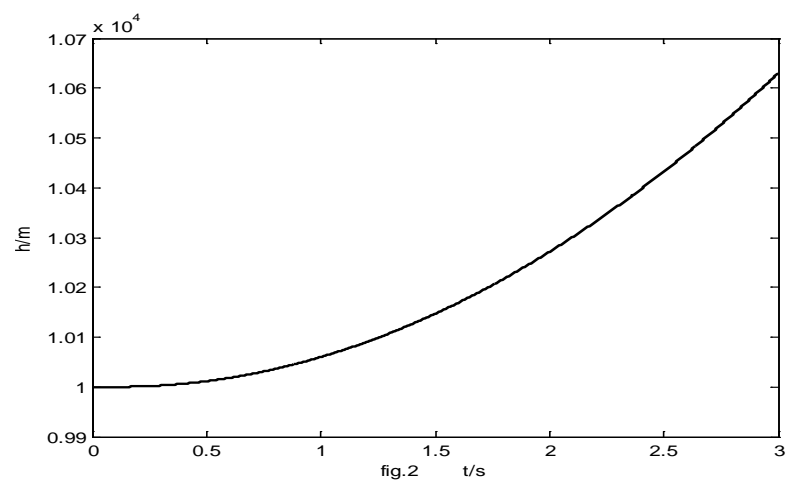

Fig. 3 Height curve 


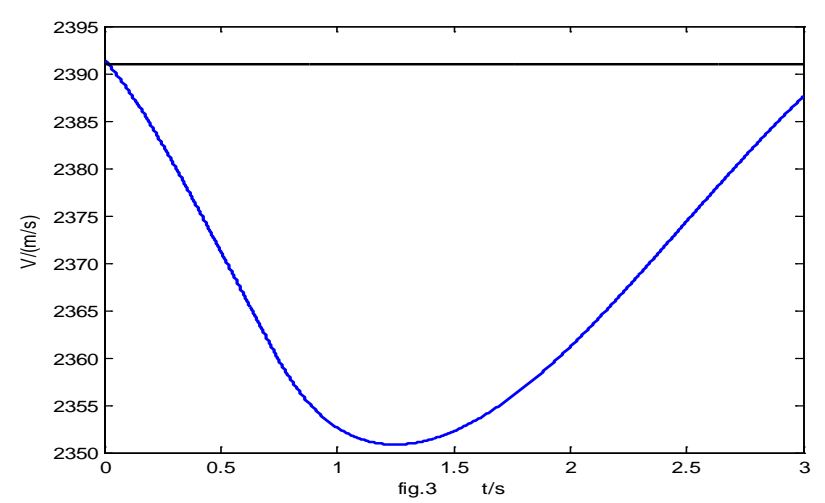

Fig. 4 Velocity curve

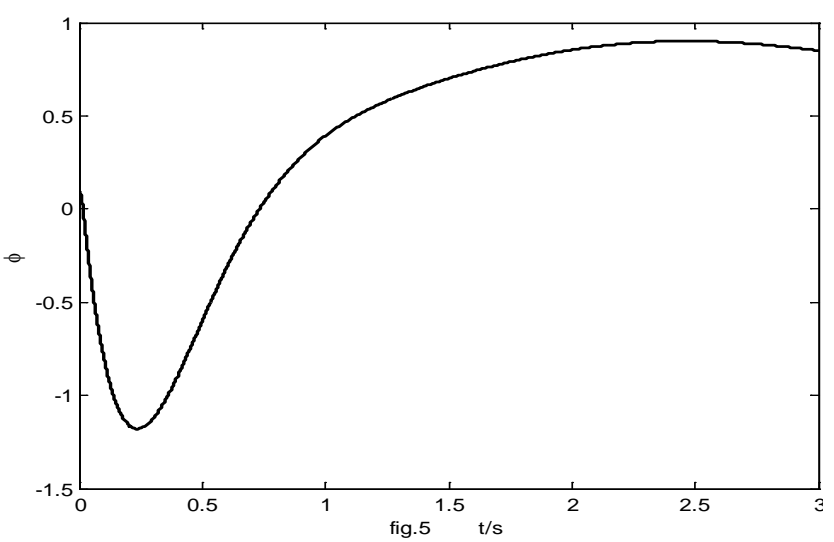

Fig. 6 Fuel supply factor curve

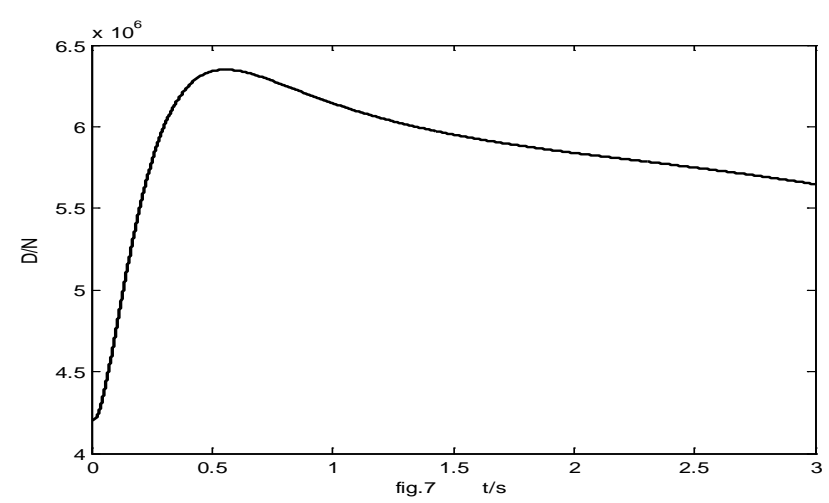

Fig. 8 Resistance curve

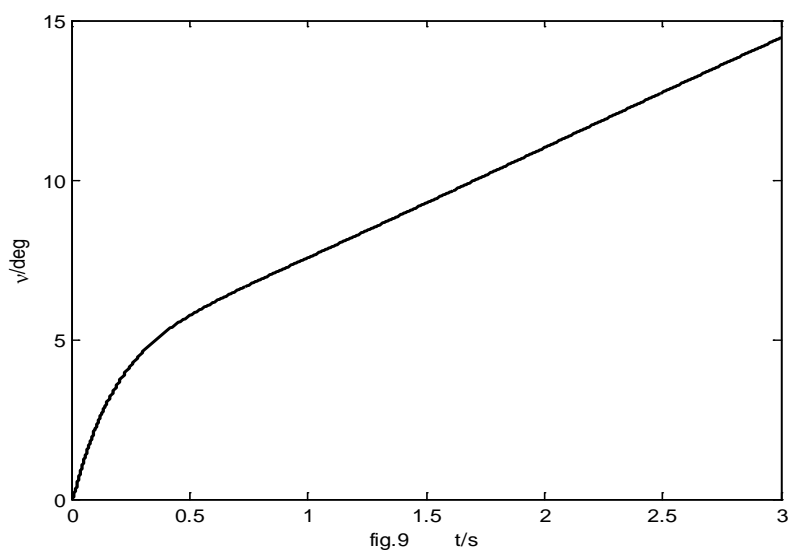

Fig. 10 Attitude angle curve

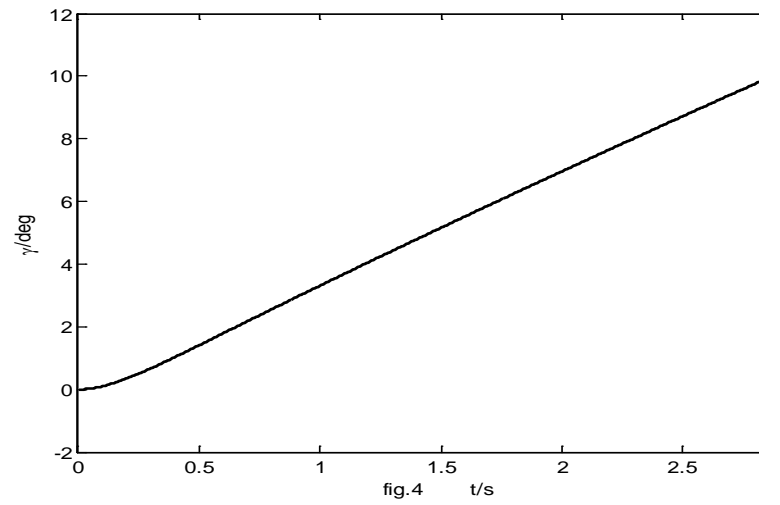

Fig. 5 Speed inclination curve

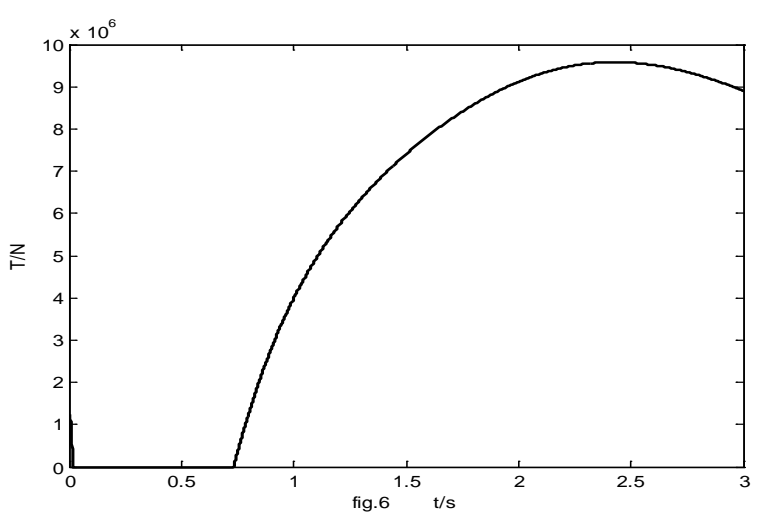

Fig. 7 Thrust curve

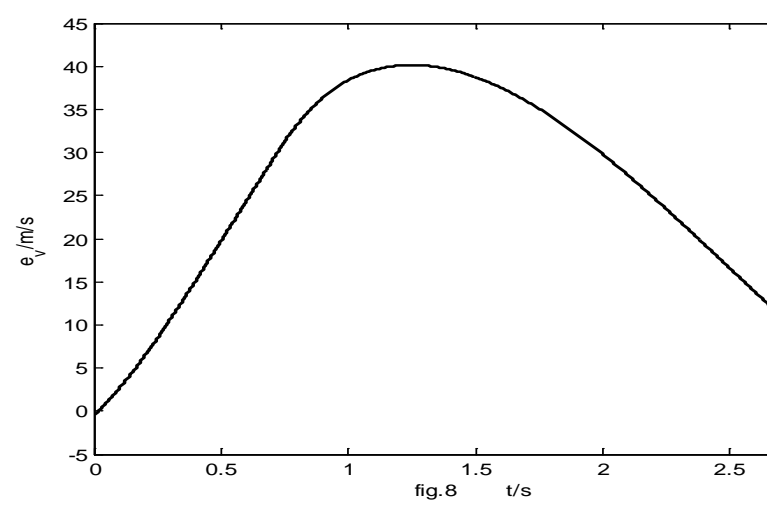

Fig. 9 Velocity error curve

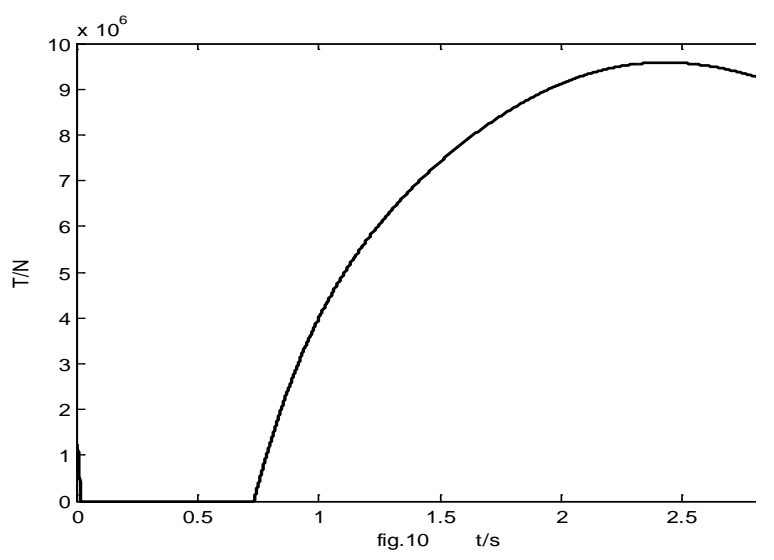

Fig. 11 Lift curve

From the several procedures and simulation curves above, We can see that under the action of the angle of attack tracking PID controller, angle of attack tends to be stable and in over damping 
state without overshoot. But without control it has overshoot in less damping state .At this time, the velocity and attitude angle are both in the slope response state, that is, uniform speed increases. And the height is in a rapid climb in the state, which climbs 450 meters in about 2.5 seconds. Overall, the open-loop model is relatively stable and similar to the general supersonic missile.

\section{Conclusions}

According to the nonlinear model of pitch channel of a hypersonic vehicle, in this paper, a class of improved PID controller is constructed to improve the stability of hypersonic vehicle by introducing the angle of attack and the pitch angle rate, which increases the system damping. And the digital simulation verifies the correctness and validity of this method.

\section{References}

[1]Snell, Nonlinear Dynamic-Inversion Flight Control of Super maneuverable Aircraft, University of Minnesota,1991.

[2]R. R. da Costa, Q. P. Chu, J. A. Mulder, Re-entry flight controller design using nonlinear dynamic inversion [A], In: AIAA Guidance, Navigation, and Control Conference and Exhibit [C], Montreal: AIAA, 2001-4219

[3]Juliana S., Chu Q. P., Mulder J. A., etal. Flight control of atmospheric re-entry vehicle with non-linear dynamic inversion[A], //In: AIAA Guidance, Navigation, and Control Conference and Exhibit [C], Providence: AIAA, 2004, 2004-5330.

[4]Mickle MC and Zhu JJ, Bank-to-turn roll-yaw-pitch autopilot design using dynamic nonlinear inversion and PD eigenvalue assignment [A], In: Proceedings of the American Control Conference[C], Chicago: IEEE, 2000:1359-1364.

[5]Zhu JJ, Banker D, and Hall CE, X-33 Ascent Flight Control Deign by Trajectory Linearization A Singular Perturbation Approach [A], In: AIAA Guidance, Navigation, and Control Conference and Exhibit, Denver: AIAA, 2000: 1-19.

[6]Zhu JJ, and Huizenga A, A type two trajectory linearization controller for a Reusable Launch Vehicles -- A Singular Perturbation Approach[A], In:AIAA Atmospheric Flight Mechanics Conference and Exhibit[C], Providence: AIAA, 2004: 1-16.

[7]Bevacqua T, Best E, Huizenga A, and et all, Improved trajectory linearization flight controller for Reusable Launch Vehicles [A], In: 42nd AIAA Aerospace Sciences Meeting and Exhibit, Reno: AIAA, 2004:1-16.

[8]Marwan Bikdash, Ken Sartor, Abdollah Homaifar, Fuzzy guidance of the shuttle orbiter during atmospheric reentry [J], Control Engineering Practice, 1999 (7): 295-303

[9]Wu S F, Engelen J H,BabuSka R. Intelligent flight controller design with fuzzy logic for atmospheric reentry Vehicle. AIAA,Aerospace Sciences Meeting and Exhibit,2000.

[10]Austin K J, Jacobs P A, Application of Genetic Algorithms to Hypersonic Flight Control [A]. IFSA World Congress and 20th NAFIPS International Conference Austrilia,2001, 2428-2433.

[11]Elmar M. Wallner, Klaus H. Well, Attitude control of a reentry vehicle with internal dynamics [A], In: AIAA Guidance, Navigation, and Control Conference and Exhibit [C], Monterey: AIAA, 2002-4647

[12]S. F. Wu, C. J. H. Engelen, R. Babuska, Fuzzy logic full-envelope autonomous flight control for an atmospheric re-entry spacecraft [J], Control Engineering Practice, 2003 (11): 11-25 